\title{
La economía mundial amesetada: algunas reflexiones a ocho años de la última crisis financiera global
}

The stagnant world economy: some reflections at eight years of the last global financial crisis

\author{
Flavio Ernesto Buchieri \\ Universidad Nacional de Villa María \\ y Universidad Tecnológica Nacional, \\ Facultad Regional Villa María, Argentina. \\ E-mail: flavio.buchieri@gmail.com
}

Fecha de recepción: 03/08/2016 Fecha de aceptación: 12/12/2016

Palabras clave

- crisis financiera

- crecimiento económico

- mundo multipolar

Keywords

- financial crisis

- economic growth

- multipolar world

\section{Resumen}

La economía mundial aún no se recupera totalmente luego de la ocurrencia de la pasada Crisis Financiera en 2008, que comenzó en Estados Unidos, se acentuó en la Euro Zona y se extendió por todo el planeta. Si a estos aspectos se le suman las tensiones económicas y geopolíticas que surgen de un mundo multipolar, las perspectivas a corto plazo no serán las esperables a partir del análisis histórico de las series contempladas. Analizar la dinámica de estos aspectos constituye el objetivo central de este artículo.

\footnotetext{
Abstract

The world economy is not yet fully recovered yet after the occurrence of the last financial crisis in 2008, wich began in United States, was accentuated in Euro Zone and spread across the globe. If we add geopolitical and economic tensions arising from a multipolar world, short term perspectives are not expected from historical analysis of statistics series. Analizing the dynamic of these aspects is the main goal of this paper.
} 


\section{Introducción: el contexto para el análisis}

Si bien gran parte de los medios de comunicación mundiales expresa que el planeta está superando la Crisis de 2008, es cierto expresar que la economía global lejos está de haber recuperado las principales tendencias económicas que se observaban antes del estallido de las hipotecas de baja calidad en los Estados Unidos. En ese marco se debate también en qué etapa se encuentra el capitalismo actual, tanto como sistema de producción como, al mismo tiempo, paradigma de interacción de agentes con motivaciones morales y éticas.

El factor que gatilla la crisis es el atentado a las Torres Gemelas de 2001, poniendo en evidencia la vulnerabilidad de los Estados Unidos no sólo como imperio dominante sino como garante del capitalismo mundial (Sela, 2012). La crisis política y de legitimidad global de dicho país afecta a la tasa de crecimiento mundial, lo cual genera una imperiosa necesidad por recuperar el mismo llevando a Alan Greenspan, presidente de la Reserva Federal, a bajar las tasas de interés para estimular la producción y la inversión a comienzo de los años 90.

Sumado a lo anterior, es opinión dominante en muchos analistas geopolíticos que las guerras ocurridas en los últimos quince años son un clara consecuencia de la imperiosa necesidad de Estados Unidos por recuperar el dominio de la hegemonía mundial y el protagonismo militar, produciendo incrementos sustanciales en el gasto público americano y su correlato en enormes déficit comerciales, financiados con los superávits comerciales de China e India, aspectos que han conducido a la debilidad del dólar a nivel mundial. La consecuencia de este proceso fue el recalentamiento de la economía mundial (Laffaye, 2009). Así, a mediados de 2007 la Reserva Federal comenzó a subir la tasa de interés, precipitando el desapalancamiento y la falta general de las coberturas que sobre la producción extensiva de derivados financieros se habían exponenciado sin regulación en numerosos bancos de inversión. La crisis estalla no en el mundo en desarrollo sino en el centro de las finanzas mundiales (Izquierdo y Talvi, 2009).

Por supuesto, la suba de la tasa de interés es un fenómeno que contribuye a la crisis producto de que el sistema financiero - particularmente, el segmento americano, un gran jugador a nivel mundial - había generado incentivos para el desarrollo de instrumentos financieros sin regulación. Cuando la tasa de interés sube los deudores comienzan a tener problemas para el repago de sus deudas y la falta de pago comienza a generar insolvencia en algunas entidades que inicialmente soportan la crisis. Cuando son muchos los deudores que no pueden pagar sus deudas son, en consecuencia, muchos los bancos que no pueden cubrir su cartera de préstamos (Buchieri, 2008, 2009 y 2011). Así, la insolvencia de los bancos puede generar una quiebra individual 0 generalizada. A través de la historia de la humanidad se ha asistido a estos problemas, pero con el desarrollo y la maduración de capitalismo las crisis parecen ser más recurrentes y más profundas por lo que caída de un gran banco de inversión como Lehman Brothers es la que genera la señal de alerta y a partir de ahí la crisis se esparce por todo el sistema financiero, generando un periodo de recesión a nivel mundial.

A pesar de la gravedad de la crisis, los bancos empezaron a sanearse y pagaron un precio muy alto por ello, sumado al fuerte proceso de ayuda financiera que recibieron como de intervención pública para su sostenimiento. Esto generó enorme problemas sociológicos en la sociedad americana, que se dividió entre aquellos que propugnaban que los bancos fueran castigados porque habían generado este proceso de excesivo apalancamiento financiero para expandir sus políticas de préstamos destinados a consumo e inversión versus los que pensaban que si los bancos caían cae toda la economía ya que los mismos, a pesar de su accionar negligente, cumplen una función importante en una sociedad capitalista porque canalizan el ahorro hacia la inversión. En la opinión de esta corriente, 
si los bancos caen interrumpirán ese proceso y, por ende, la tasa de crecimiento de la economía bajará precipitadamente. Es de destacar que, ya sea por lobby o por sensatez en la clase política americana, primó la conciencia y la sensatez por el equilibrio a largo plazo. A pesar de la fuerte política de saneamiento y capitalización que les fue exigida, hoy en día están más saneados y capitalizados que diez años atrás.

Ahora bien, la crisis que comenzó en Estado Unidos luego se propagó a Europa particularmente por el canal financiero vía suba de tasas de nivel ya mundial (CEPAL, 2009). Lo que inicialmente fue una crisis financiera de descalce entre activos y pasivos bancarios en Estados Unidos termina impactando en la Eurozona, particularmente, por los fuertes desequilibrios macroeconómicos que tenían algunos países europeos, esto es, diferentes posiciones relativas en términos de productividad, déficitfiscal y alto nivel de endeudamiento. Se asiste así a la «Europa de las dos velocidades», una región de países con alta productividad y buenos indicadores macroeconómicos (Alemania, Francia los países Nórdicos) y la que algunos analistas han denominado como «Periferia de Europa», o sea, la región integrada por países como España, Portugal, Irlanda e Italia que tienen un contexto macroeconómico distinto con déficit fiscal crónico, alto endeudamiento, baja productividad, un comercio muy concentrado entre los países europeos y términos de intercambio sin expectativas de cambio.

Conviene efectuar una pequeña intervención a la importancia, rol e incentivos en la constitución y mantenimiento de la Eurozona. Cuando finaliza la Segunda Guerra Mundial, la Convención de Yalta — que congrega a Roosevelt, Churchill y Stalin - permite asignar 0 dividir el planeta en tres áreas o regiones de inferencia, intervención o preferencia: América Latina -bajo la influencia de Estados Unidos-, África — bajo control europeo- y Asia — con influencia soviética-. En ese marco y tras los costos de reparación y de legitimidad internacional de Europa es que surge lo que cincuenta años después terminaría siendo la Eurozona. Básicamente, puede ser concebida como, en las últimas tres décadas, la ambición de liderazgo de la región europea, que pasa de Inglaterra hacia Alemania. Es por ello que cuando vemos a la Eurozona bajo la fuerte influencia de Alemania en comendar el proceso de ordenamiento territorial, monetario, comercial y hasta fiscal que surge en los últimos dos años - es porque Alemania tiene fines imperiales. Los contribuyentes alemanes han tenido que asignar muchos recursos propios para mantener tener a flote la Eurozona. Esto último hoy en día está fuertemente en discusión con lo cual, todas las medidas que se tomaron fueron para dar oxígeno a los países afectados para que estos pudieran corregir los desequilibrios que tenían, en un contexto donde medidas tradicionales pueden llegar a beneficiar a los países que en otros contextos no se producirían (Fernandez-Arias, Powell y Rebucci, 2009).

Un detalle importante a tener en cuenta a partir del análisis antes realizado es que la integración de la Eurozona implicaba pérdida de soberanía monetaria, esto es, resignar la moneda propia en pos de la moneda común, el Euro. Este fue al aspecto distintivo de Inglaterra, que integró la Eurozona pero no perdió su moneda. Tanto por razones de peso como la importancia de Londres como gran centro financiero mundial, como del hecho de que cuando los países pierden su moneda están renunciando a disponer de un instrumento clave para corregir algunos desequilibrios macro como lo es la devaluación de la misma frente a divisas internacionales, los países afectados debieron proceder a corregir los mismos con medidas costosas en términos sociales como la reducción de salarios, del gasto público y del déficit fiscal, entre otras, más allá de que sus niveles de deuda sufrieron, en algunos casos, quitas de capital para dar oxígeno a estas economías en la superación de la crisis y su paso hacia una transición más estable para permanecer en la región.

Lo antes delineado es el contexto que sirve de soporte al análisis que continuaremos observando. 


\section{El mundo hoy: breve radiografía de la situación económica mundial y sus perspectivas}

A través de algunos gráficos mostraremos cómo nos encontramos hoy. El mundo se ha recuperado pero con la salvedad de que la crisis no ha sido enteramente superada. Esto acontece porque los desequilibrios que se acumularon en el pasado son de difícil corrección. Y si a ello se le agrega que a nivel mundial hay crisis de liderazgo a nivel de las grandes superpotencias, es menester destacar que la corrección macroeconómica del crecimiento mundial haya sido muy leve y atenuada por la propia dinámica de estos desequilibrios macroeconómicos (FMI, 2014).

Los datos elaborados permiten considerar que la economía mundial cae fuertemente en 2009 , asistiéndose luego a una pequeña recuperación con una estabilidad que hemos observado en los últimos años. Sin embargo, la recuperación actual está lejos del punto de partida previo al inicio de la crisis, observando diferentes situaciones para cada uno de los grupos de países que hemos tomado para poder efectuar esta consideración (véase el siguiente gráfico).

Gráfico 1. Evolución de las Tasas de Crecimiento Globales y por Regiones.

\section{En \%. Período 2007-2015}

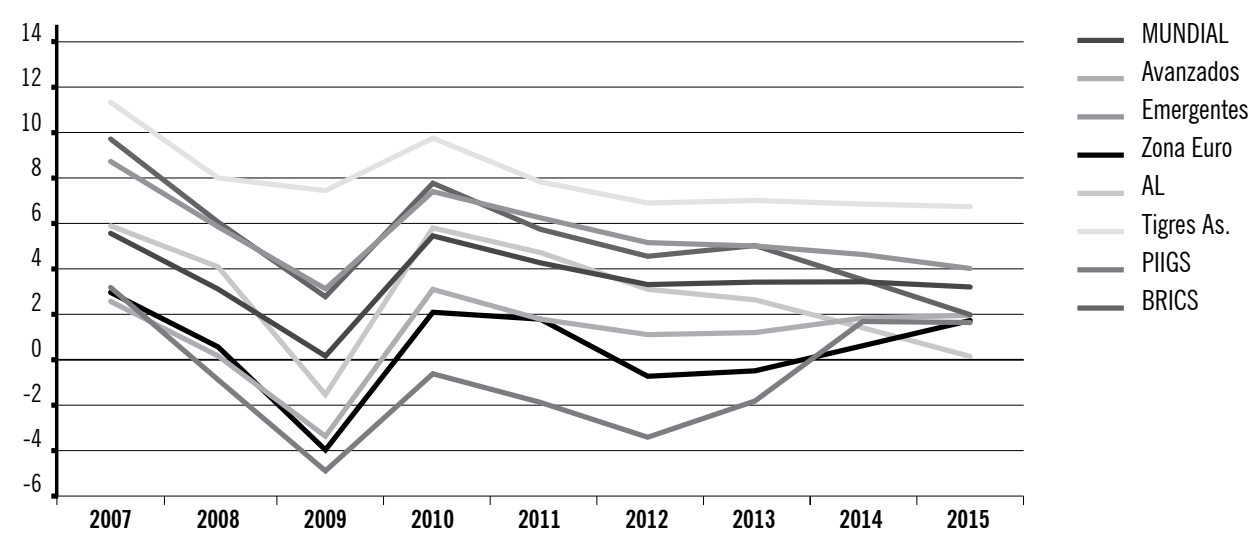

Fuente: Elaboración propia en base a Eurostat, 2016

A partir de analizar un período más amplio como es el comprendido por los años 1980-2015, se puede observar que, como se expone en el siguiente gráfico, el promedio mundial para el mismo era del 3,5\%, subió fuertemente en el período 2000-2007 —un período de gran estabilidad, "gracias» al atentado de las torres gemelas que llevó a que la
Reserva Federal de los Estados Unidos aumentara fuertemente la liquidez para estimular el crecimiento mundial-y, tras la crisis, vuelve al 3,5\%. Es decir que, en la actualidad, hemos recuperado el promedio histórico pero no estamos cerca de lo que fueron los años previos a la crisis. 
Gráfico 2. Evolución de las Tasas de Crecimiento para el promedio mundial. En \%. Período 2007-2015 y promedios por diferentes períodos

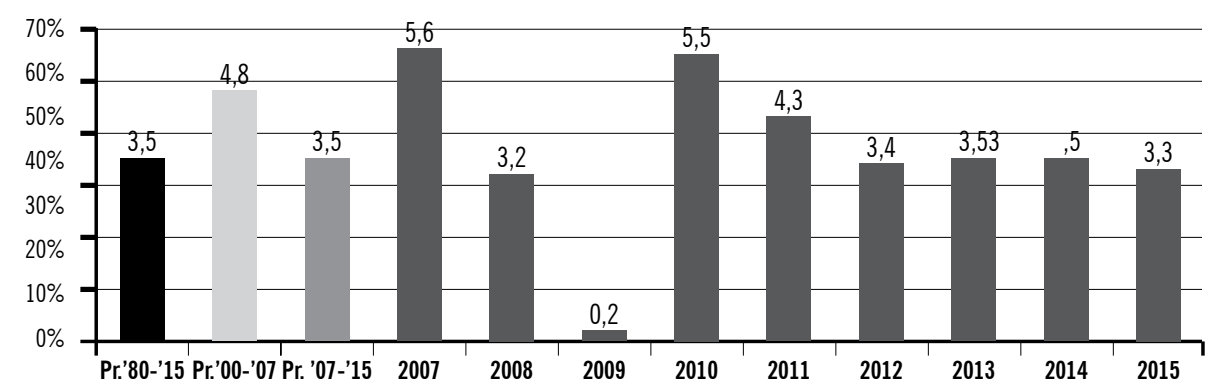

Fuente: Elaboración propia en base a Eurostat, 2016

Cuando se analiza al interior de los grupos de por supuesto, el panorama es ampliamente diverso países, América Latina registraba un 2,9\% como tasa de crecimiento del período amplia, subiendo fuertemente al 5,1\% para el subperíodo 20002007, ubicándose, hoy, en las tasas históricas. Sí, para otras regiones, con claros ganadores y perdedores antes, durante y después de la crisis, como se observa en el siguiente gráfico y que explayaremos con más detalle a continuación.

Gráfico 3. Evolución Tasas de Crecimiento Globales y por Regiones (en\%)
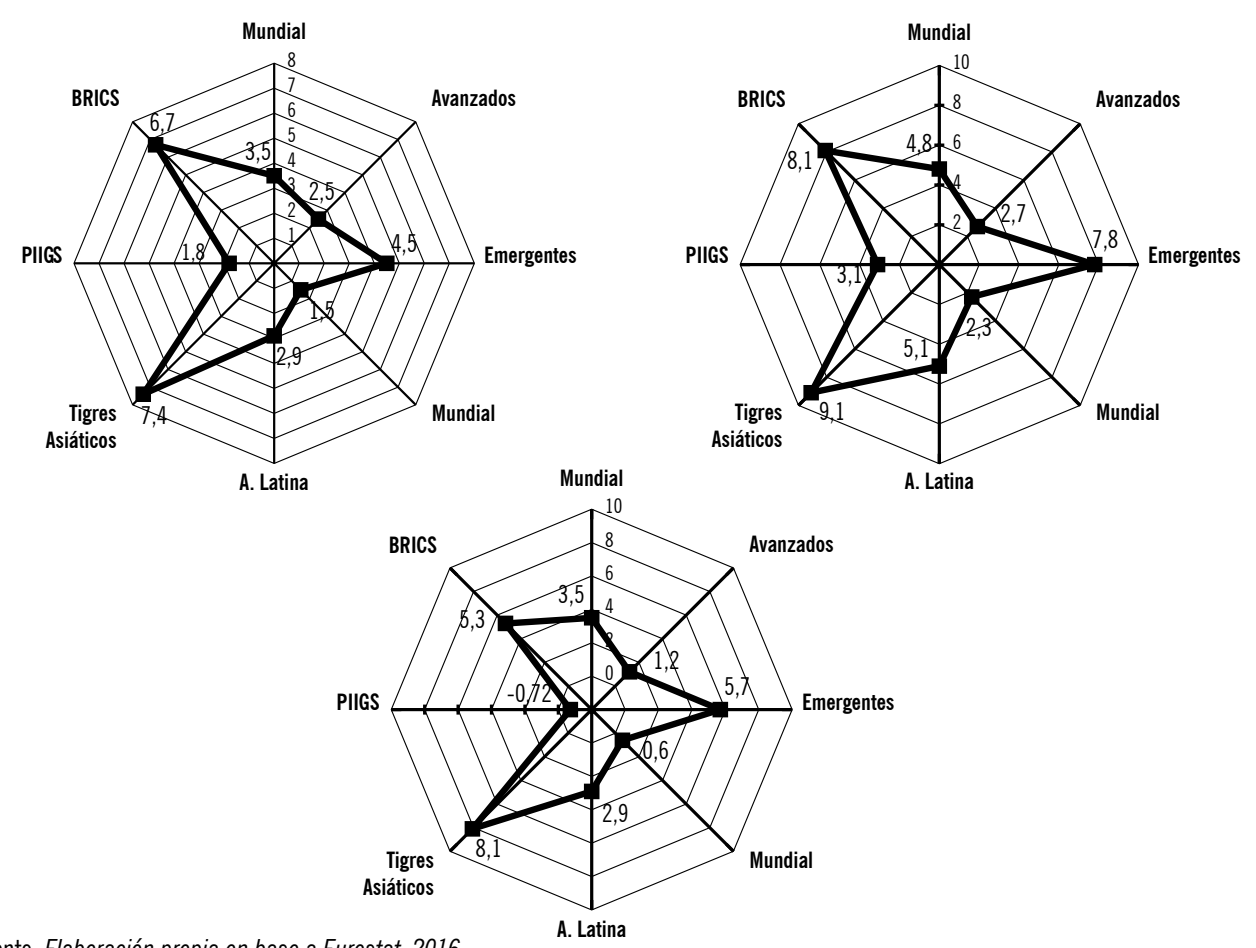

Fuente: Elaboración propia en base a Eurostat, 2016 
En términos de niveles de producción medidos por índices, tomando como base el año 2007 e igual a 100 , en la actualidad se asiste a un nivel de 129 , es decir que hoy en día no solamente hemos recuperado el punto de partida sino que el planeta está produciendo un $29 \%$ más.

Gráfico 4. Evolución del PBI Promedio Mundial.

Indice $A \tilde{n ̃ o} 2007=100$.

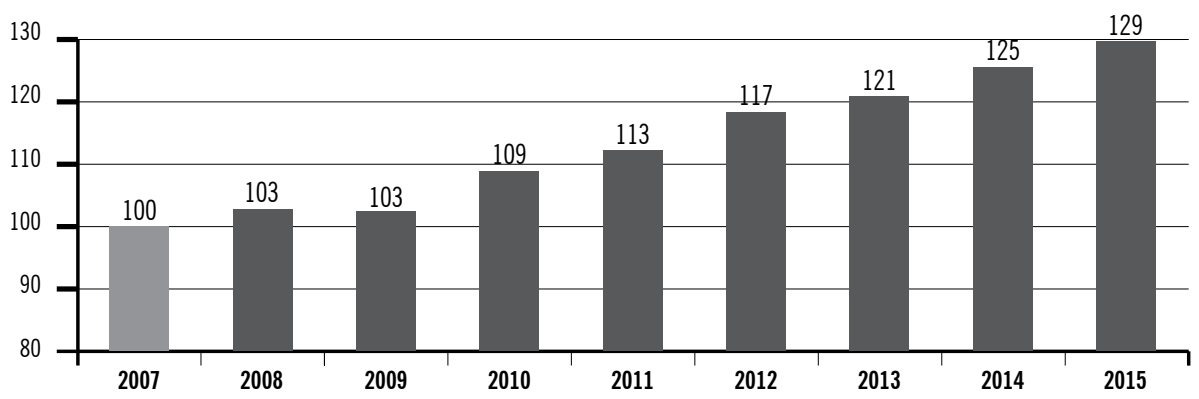

Fuente: Elaboración propia en base a Eurostat, 2016

\section{Análisis por regiones o grupos de países}

Las diferencias se observan cuando, hacia el interior del análisis global, se discrimina por grupos 0 regiones de países. Los datos convalidan los análisis, tendencias $\mathrm{y} / 0$ credos dominantes, esto es, en términos de tasas $y / 0$ niveles de crecimiento, son los países desarrollados los que más han sufrido la crisis, como se expone en el siguiente gráfico.

Gráfico 5. Evolución de las Tasas de Crecimiento para los Países Avanzados.

En \%. Período 2007-2015 y promedios por diferentes períodos-

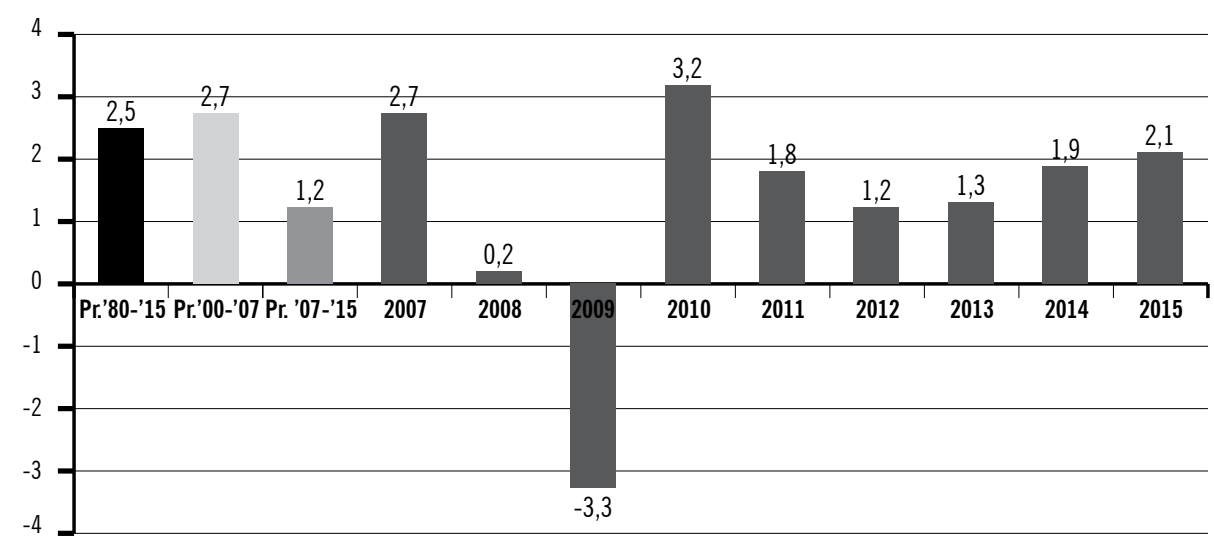

Fuente: Elaboración propia en base a Eurostat, 2016 
Estos países venían creciendo a un 2,5\% para el período histórico, cayendo a $-3,3 \%$ en el peor año de la crisis, para luego arribar a tasas estables pero más bajas que el promedio mundial, siendo que su promedio para el subperiodo 2007-2015 es más bajo que el registrado para todo el período amplio 1980-2015 y, a su vez, muy por detrás del promedio mundial: $1.2 \%$. En términos de niveles de producción, y tomando siempre al año 2007 $=100$, para el año 2005 su nivel era de 108, esto es, un 8 $\%$ más que en el año base.

\section{Gráfico 6. Evolución del PBI de los Países Avanzados.}

Indice Año 2007=100.

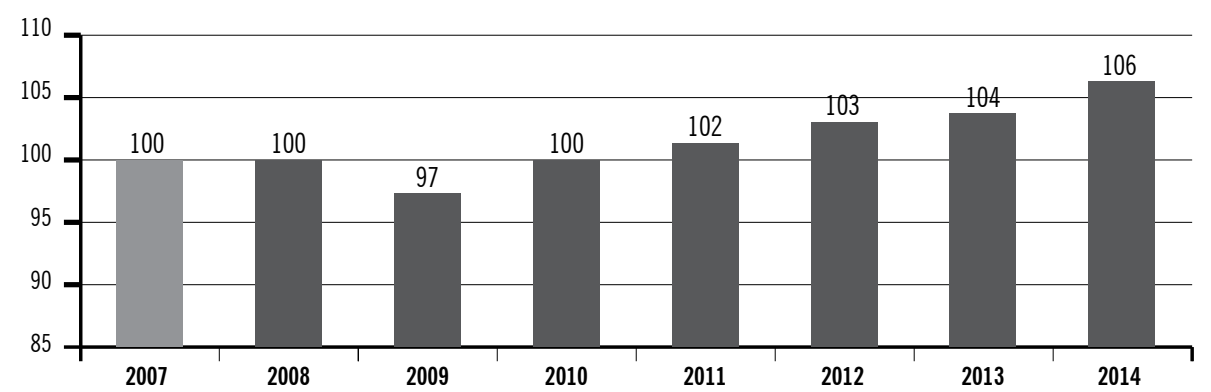

Fuente: Elaboración propia en base a Eurostat, 2016

Para los países emergentes la situación es bien de crecimiento positivo del 3,2 \%, para luego recudistinta. En el peor momento de la crisis pasaban de crecer al 8,8\% en el año 2007 a registrar una caída importante pero aún manteniendo una tasa perarse y mostrar tasas más altas que el promedio mundial y en torno al $5.7 \%$ promedio para el período 2007-2015.

Gráfico 7. Evolución de las Tasas de Crecimiento para los Países Emergentes.

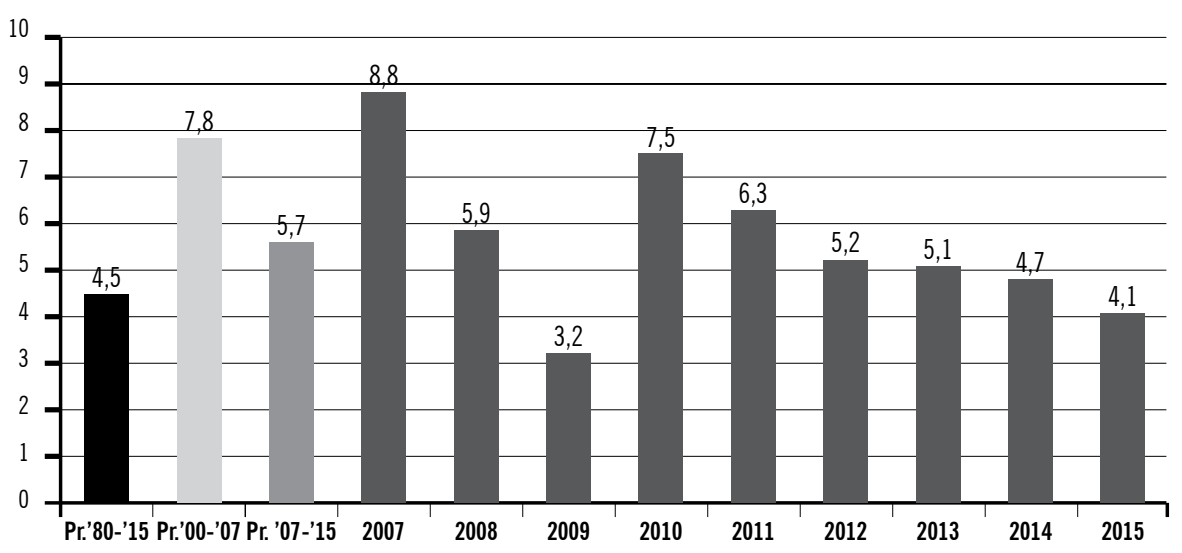

Fuente: Elaboración propia en base a Eurostat, 2016 
Por otro lado, en términos de niveles de producción, hoy producen un 49 \% más de lo que producían en el año 2007.

Gráfico 8. Evolución del PBI de los Países Emergentes.

Indice Año 2007=100

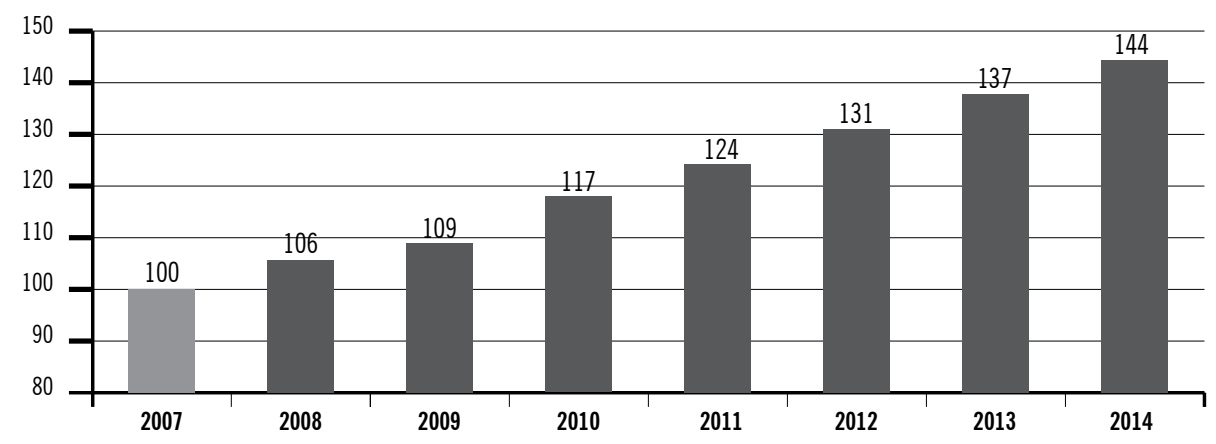

Fuente: Elaboración propia en base a Eurostat, 2016

En los países de la zona del euro su situación es aún más grave que la registrada para los países desarrollados. Sus promedios de crecimiento para el período 1980-2015 como subperíodosestán bien por debajo del promedio mundial como de los países desarrollados. Pero, a su vez, en el peor año de la crisis registran una caída del $3.9 \%$ al mismo tiempo que vuelven a asistir a registros negativos en su tasa de crecimiento para los años 2012 y 2013. Todo esto permite considerar que el promedio para el subperíodo 2007-2015 sea del orden del $0.6 \%$, el peor registro en relación al promedio mundial como del resto de los grupos 0 regiones.

Gráfico 9. Evolución de las Tasas de Crecimiento para los Países Zona Euro.

En \%. Período 2007-2015 y promedios por diferentes períodos

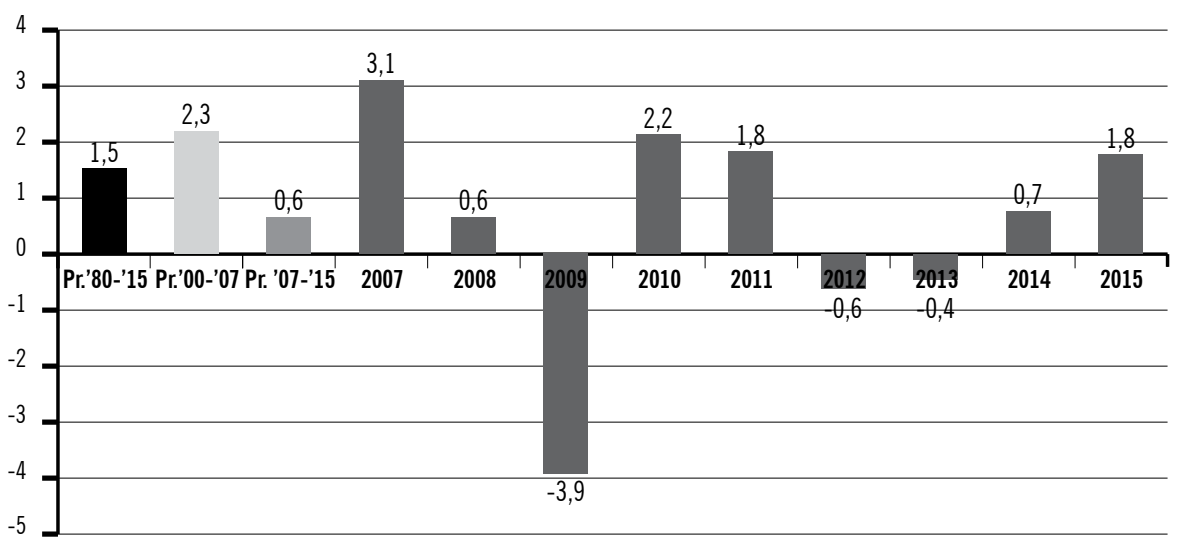

Fuente: Elaboración propia en base a Eurostat, 2016 
Cuando analizamos el nivel de producción de riqueza, en términos del año 2007 =100 en el 2015 producían lo mismo que ocho años atrás.

Gráfico 10. Evolución del PBI de los Países Zona Euro.

Indice Año 2007=100

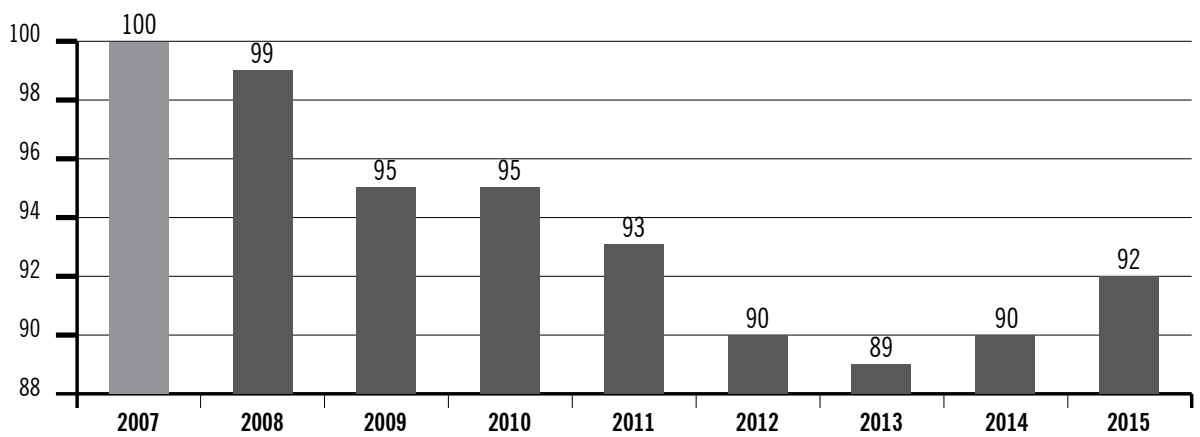

Fuente: Elaboración propia en base a Eurostat, 2016

¿Cómo ha sido el desempeño de los PIIGS, esto es, Portugal, Italia, Grecia, Irlanda y España? Estos países no venían tenían un desempeño económico tan mal en el período y/0 año previo a la crisis. Sin embargo, caen fuertemente en el año 2009 y continúan con performances negativas para los años 2011, 2012 y 2013, arribando a un promedio para el subperíodo 2007-2015 de -0.7\%.

Gráfico 11. Evolución de las Tasas de Crecimiento para los PIIGS

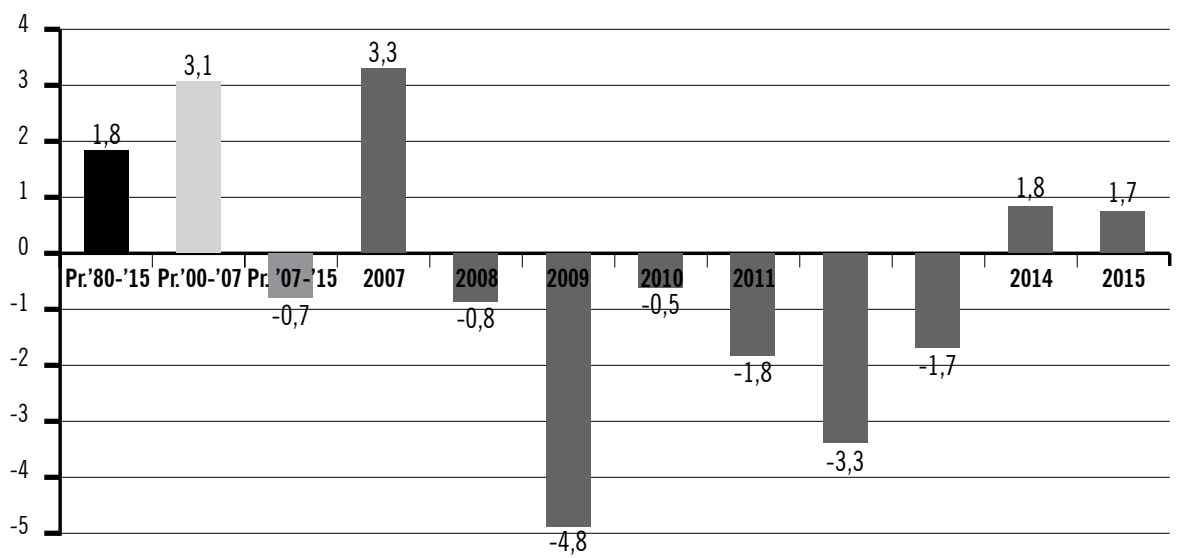

Fuente: Elaboración propia en base a Eurostat, 2016

En términos de producción de riqueza, la situación económica negativa es contundente: de producir
100 en el año 2007, hoy están muy por debajo, menos de un $10 \%$ con respecto al año base. 
Gráfico 12. Evolución del PBI de los PIIGS.

Índice Año 2007=100

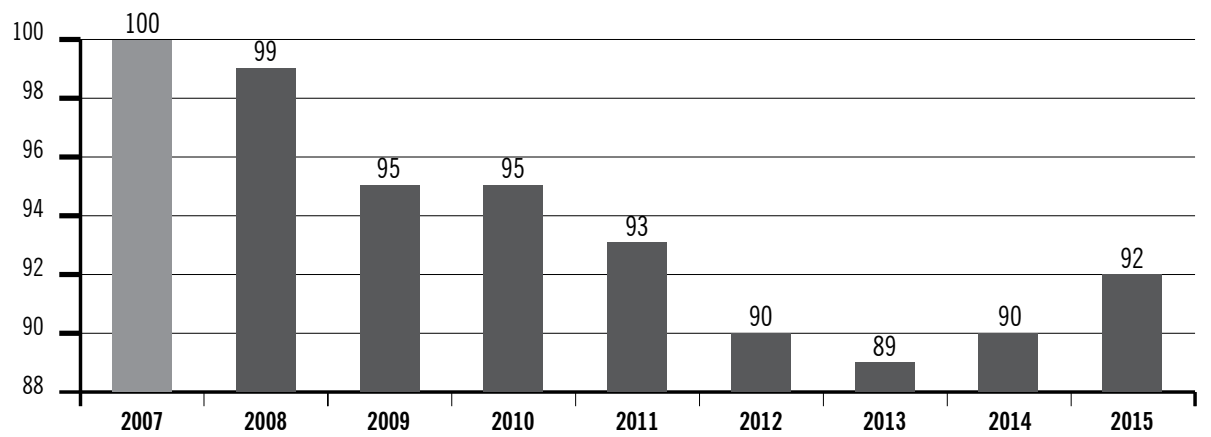

Fuente: Elaboración propia en base a Eurostat, 2016

El quinto grupo de países a analizar en América ríodo 2007-2015 del orden del $2.9 \%$ que, por cierto, Latina. En el peor año de la crisis, la Región cae a coincide con el promedio de todo el período 1980un $1,5 \%$ de crecimiento cuando el mundo cayó $3 \%$. 2015. Cuando hacemos referencia a los niveles de La recuperación posterior no ha sido consistente ni producción, en el 2015 se estaba obteniendo un 22 duradera, arribando a un promedio para el subpe- \% más que en relación con el año base.

Gráfico 13. Evolución de las Tasas de Crecimiento para America Latina.

En \%. Período 2007-2015 y promedios por diferentes períodos

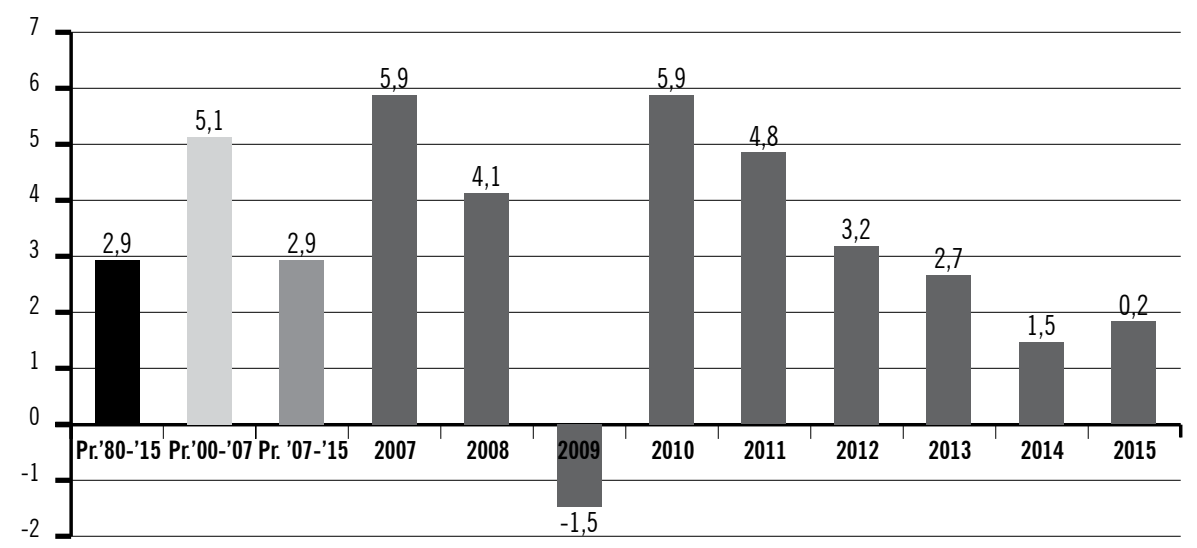

Fuente: Elaboración propia en base a Eurostat, 2016 
Gráfico 14. Evolución del PBI de los Países De América Latina.

Índice Año 2007=100

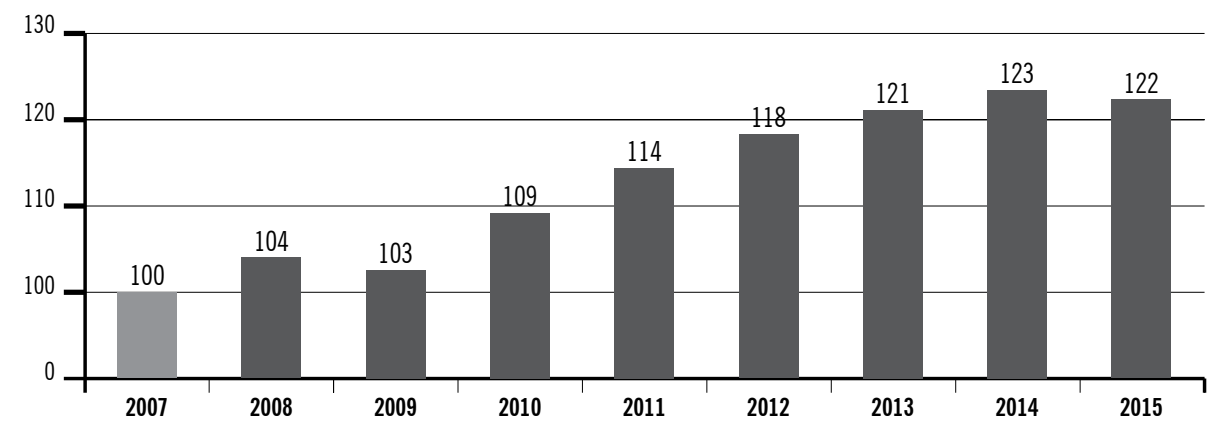

Fuente: Elaboración propia en base a Eurostat, 2016

Sin lugar a dudas que los Tigres Asiáticos incluido China — son los que mejor desempeño han mostrado antes, durante y después de la ocurrencia de la crisis, que tuvo acotado impacto en el peor año de la misma. La región se ha estabilizado en torno a una tasa de crecimiento promedio del $8 \%$ para el subperíodo 2007-2015. En términos de niveles de producción, en el pasado año 2015 el grupo producía un 77 \% más que en relación con el año base.

Gráfico 15. Evolución de las Tasas de Crecimiento para los Tigres Asiáticos.

En \%. Período 2007-2015 y promedios por diferentes períodos

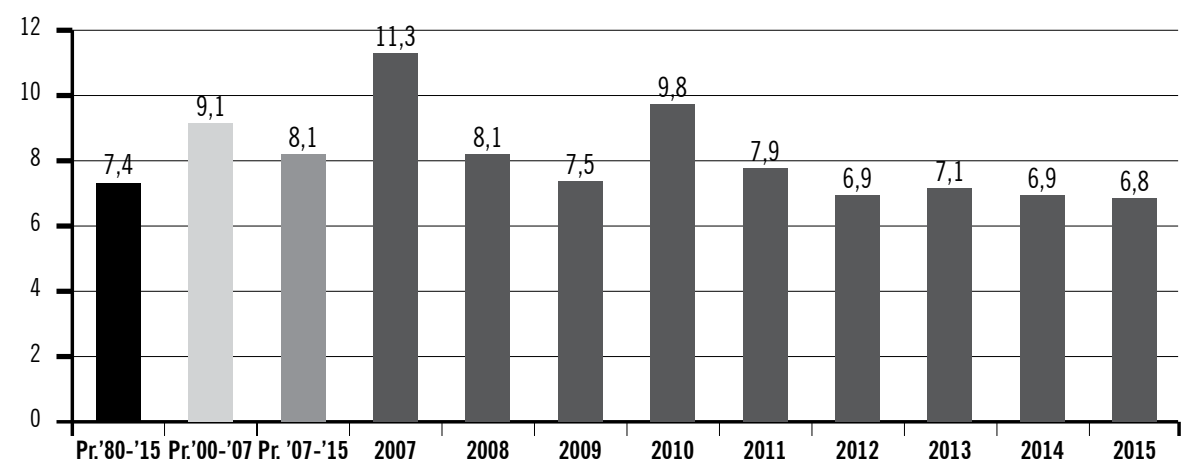

Fuente: Elaboración propia en base a Eurostat, 2016 
Gráfico 16. Evolución del PBI de los Tigres Asiáticos.

Indice Año 2007=100

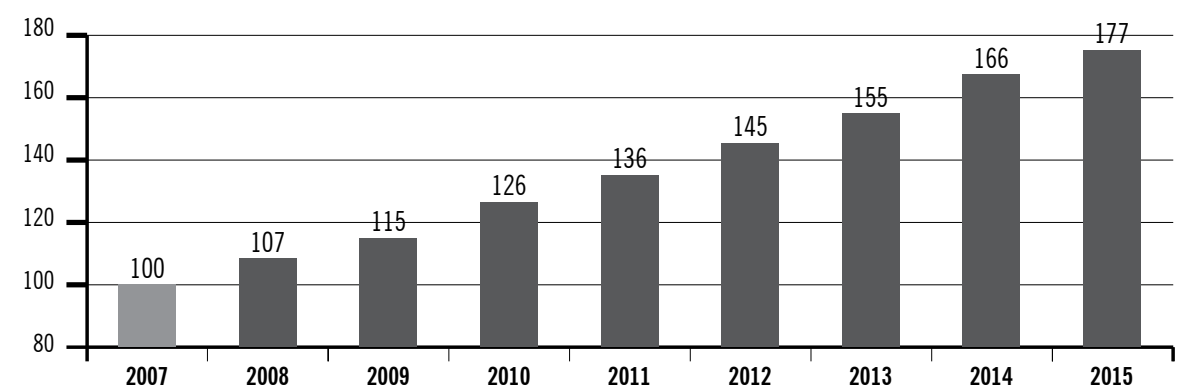

Fuente: Elaboración propia en base a Eurostat, 2016

El último grupo a analizar es el de los BRICS integrado por Brasil, Rusia, India, China y Sudáfrica-, hoy en día en fuerte cuestionamiento a su cohesión interna por el deficiente crecimiento y/0 situación institucional en Brasil, la mala performance de Rusia, afectada por la caída de los precios del petróleo, e India con tendencias hegemónicas en relación con China. El grupo evidenció una buena evolución económica en los últimos años — por debajo de los resultados arrojados por Ios Tigres Asiáticos - aunque pasaron de crecer al 9,7\% en 2007 a un nivel de un poco más del 2 $\%$ en el pasado 2015, arrojando un promedio para el subperíodo 2007-2015 del orden del $5.3 \%$. En cuanto a la producción agregada de riqueza, hoy están produciendo un $45 \%$ más en relación con el año base.

Gráfico 17. Evolución de las Tasas de Crecimiento para los BRICS.

En \%. Período 2007-2015 y promedios por diferentes períodos

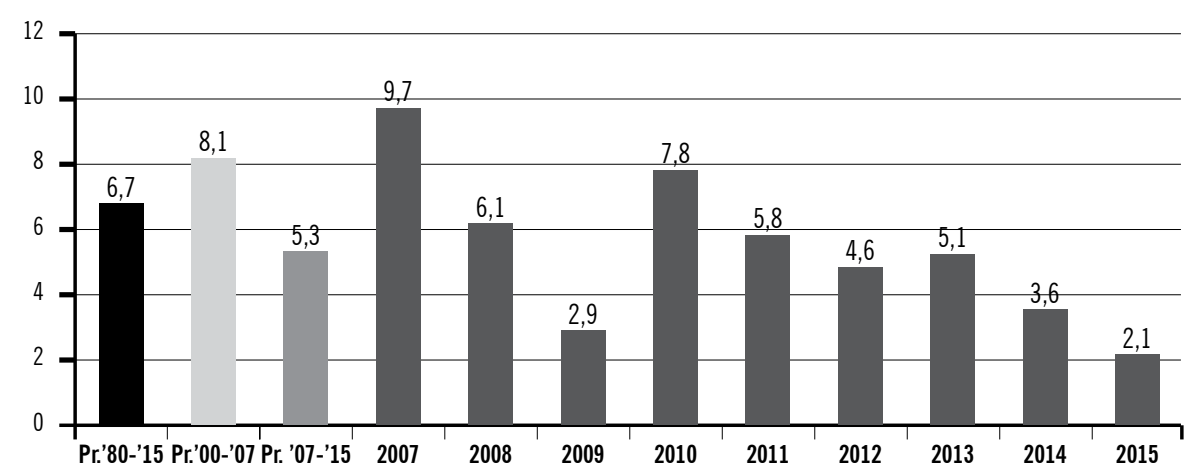

Fuente: Elaboración propia en base a Eurostat, 2016 
Gráfico 18. Evolución del PBI de los BRICS.

Indice Año 2007=100

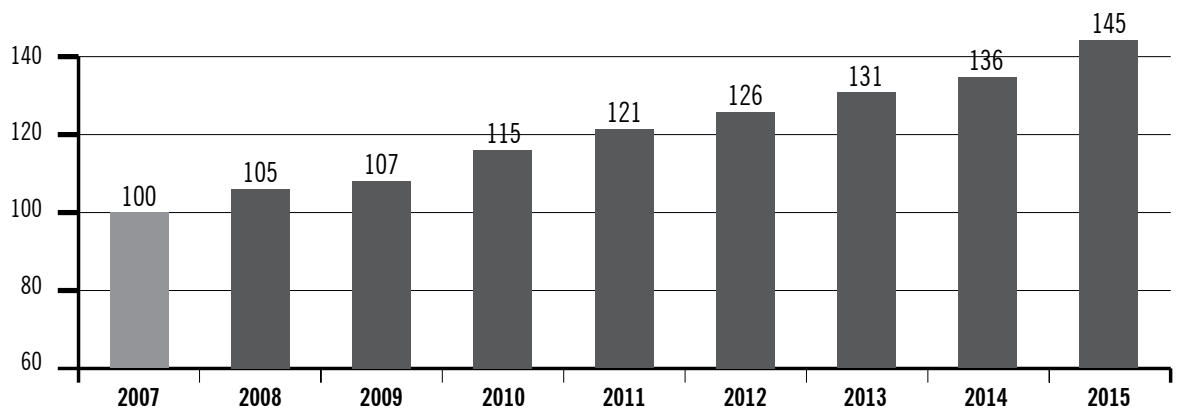

Fuente: Elaboración propia en base a Eurostat, 2016

\section{Evaluación y perspectivas a mediano plazo}

A ocho años del inicio formal de la crisis, es obvio que el crecimiento mundial todavía se encuentra amesetado en un nivel bajo en relación con el promedio de los últimos veinticinco años. Si a ese contexto le sumamos la crisis de liderazgo de los Estados Unidos como superpotencia mundial, la proliferación de conflictos regionales - particularmente, en el cercano Oriente - más la incertidumbre - hoy acotada - del impacto y los costos de la salida de Gran Bretaña de la Eurozona, será claro que, en el corto plazo, las expectativas por recuperar tasas de crecimiento más altas están congeladas.

Los países que están más afectados por la crisis — los PIIGS — están sobreviviendo en un marco donde la corrección de desequilibrios macroeconómicos no es gratis. Los ciudadanos alemanes están financiando la ayuda pero también lo están financiando los ciudadanos de otros países con reducción de subsidios, salarios, presupuestos públicos, presupuestos educativos, jubilaciones y pensiones, entre otros, afectando las tendencias de crecimiento de estos últimos países. Por otro lado, los commodities primarios van a continuar con tendencia decreciente en los próximos años si es que la correlación inversa con el dólar se sigue manteniendo a futuro. La soja probablemente se recupere en el corto plazo aunque se construyen malas perspectivas para los precios de los hidrocarburos. Esto último afecta a Argentina y a Colombia. Y si a ellos se le suma que los flujos financieros se han reducido en nuestra región, será también claro que el efecto combinado de todo lo antes expuesto implica un fuerte proceso de desaceleración económica para casi todos los países de América Latina (Heroles Cardozo, 2014).

Por otro lado, los hechos históricos de los últimos años permiten observar que, en el proceso de corrección macroeconómica a nivel mundial —uno de los principales impactos colaterales de la crisis - las economías emergentes -y, especialmente, los tigres asiáticos - han demostrado que aun con tasas de crecimiento alto estos países no pueden compensar, particularmente, la reducción de la tasa de crecimiento de los Estados Unidos (Ocampo, 2009 y 2011). En ese marco, las agresivas políticas de estímulos monetarios que llevó a cabo tanto la Reserva Federal como el Banco Central Europeo no han fortalecido las expectativas de recuperación económica (la famosa «trampa de la liquidez» que postulaba Keynes está en plena vigencia), con lo cual, y particularmente, el proyecto de Reforma Fiscal de los países de la zona euro genera más presiones recesivas. Todo esto obliga a que haya un fortalecimiento de la coordinación y cooperación de las políticas económicas a nivel mundial. 
En el caso particular de la Eurozona, Alemania está imponiendo un nuevo sistema de incentivos a la racionalidad intertemporal en términos fiscales; por ende, Alemania no es solamente la que establece cuál es la relación entre el euro y el precio de las divisas, sino que también Alemania va a comenzar a definir y establecer cómo se establece la política fiscal a nivel de la Eurozona. Es esta situación la que genera mayor presión en las correcciones de la actividad económica hacia la baja en los países afectados con lo cual, si a ello se le suman los problemas sociales recientes como la inmigración de Cercano Oriente - no será descabellado pensar que haya «nuevos Brexits» a la vuelta de la esquina.
¿Cuáles son las perspectivas económicas a futuro? Pues, la mayor parte de las estimaciones indican que en 2005 el mundo creció al 2,7 \% y se va mantener casi con una muy leve recuperación en torno al 2,8 \% -3 \% para este año y el que viene. Por otro lado, la inflación está relativamente contenida en torno al $3 \%$. A nivel de las principales regiones, América Latina no tendrá una buena performance, tanto desde el punto de vista del aumento de su PBI como de su nivel de inflación. El siguiente gráfico muestra la situación para el promedio mundial y algunas regiones en términos de inflación y aumento del PBI para los años 2016 y 2017.

Gráfico 19. Perspectivas de crecimiento e inflación.

\begin{tabular}{|c|c|c|c|c|c|c|}
\hline & \multicolumn{3}{|c|}{ \% CRECIMIENTO PIB } & \multicolumn{3}{|c|}{ INFLACIÓN } \\
\hline & 2015 & 2016 & 2017 & 2015 & 2016 & 2017 \\
\hline Mundo & 2,7 & 2,8 & 3,0 & 2,0 & 3,1 & 3,0 \\
\hline OCDE & 1,9 & 2,0 & 2,1 & 0,5 & 1,5 & 2,1 \\
\hline EE.UU. & 2,4 & 2,3 & 2,4 & 0,1 & 1,5 & 2,3 \\
\hline Japón & 0,7 & 0,9 & 0,7 & 0,8 & 0,4 & 1,7 \\
\hline Zona Euro & 1,6 & 1,6 & 1,7 & 0,1 & 0,5 & 1,4 \\
\hline Rusia & $-3,8$ & $-1,0$ & 1,2 & 7,8 & 15,2 & 8,1 \\
\hline Latinoamérica & $-0,2$ & $-0,1$ & 2,1 & 13,5 & 17,3 & 10,7 \\
\hline Asia - Pacífico & 3,4 & 3,6 & 4,0 & 2,4 & 2,9 & 3,2 \\
\hline China & 6,9 & 6,4 & 6,0 & 1,5 & 1,9 & 2,2 \\
\hline India & 7,3 & 7,4 & 7,4 & 5,1 & 5,3 & 5,4 \\
\hline
\end{tabular}

Fuente: Elaboracion propia en base a BNP Paribas (2015)

En los países de la Eurozona y de Europa del este la performance económica que se observa es de bajo crecimiento (con un rango de $-1 \%$ al $4 \%$ y promedio del $1.6 \%$ para la Eurozona) y baja inflación (entre $-0.5 \%$ y $2 \%$, con un promedio del orden de $1 \%$ para la misma región), según los países.
Sí, hay preocupación por la tasa de paro o Desempleo que asume valores muy altos en relación con el mismo grupo de países, del 20 \% para España como bajos y en torno al $4 \%$ para Alemania, con un promedio en torno al $10 \%$. 
Gráfico 20. Perspectivas de crecimiento e inflación.

Eurozona y Europa del Este.

\begin{tabular}{|c|c|c|c|c|c|c|c|}
\hline \multirow[t]{2}{*}{ PAíS/ÁREA } & \multirow{2}{*}{$\begin{array}{r}\text { TASA PARO } \\
2015 \\
\end{array}$} & \multicolumn{3}{|c|}{ \% CRECIMIENTO PIB } & \multicolumn{3}{|c|}{ INFLACIÓN } \\
\hline & & 2015 & 2016 & 2017 & 2015 & 2016 & 2017 \\
\hline Alemania & 4,3 & 1,7 & 1,7 & 1,7 & 0,1 & 0,6 & 1,6 \\
\hline Austria & 5,9 & 0,8 & 1,3 & 1,5 & 0,8 & 1,5 & 1,9 \\
\hline Belgica & 7,9 & 1,3 & 1,4 & 1,5 & 0,6 & 1,3 & 1,7 \\
\hline Dinamarca & 5,9 & 1,2 & 1,5 & 1,8 & 0,2 & 0,7 & 1,5 \\
\hline España & 20,5 & 3,2 & 2,5 & 2,7 & $-0,5$ & 0,2 & 1,5 \\
\hline Finlandia & 9,4 & 0,2 & 0,6 & 1,3 & $-0,2$ & 0,4 & 1,1 \\
\hline Francia & 10,2 & 1,1 & 1,3 & 1,5 & 0,1 & 0,4 & 1,1 \\
\hline Grecia & 24,6 & $-0,4$ & $-1,0$ & 1,5 & $-1,1$ & 0,4 & 1,4 \\
\hline Holanda & 6,5 & 2,0 & 1,9 & 1,8 & 0,2 & 0,7 & 1,4 \\
\hline Irlanda & 8,8 & 6,6 & 3,8 & 3,7 & 0,0 & 0,6 & 1,3 \\
\hline Italia & 11,5 & 0,7 & 1,1 & 1,2 & 0,1 & 0,3 & 1,0 \\
\hline Polonia & 6,9 & 3,6 & 3,2 & 2,9 & $-0,7$ & 0,5 & 3,2 \\
\hline Portugal & 12,2 & 1,0 & 1,5 & 1,7 & 0,5 & 0,5 & 1,1 \\
\hline Reino unido & 5,1 & 2,2 & 2,1 & 2,1 & 0,0 & 0,6 & 1,7 \\
\hline Suecia & 7,0 & 3,6 & 3,0 & 2,7 & 0,7 & 0,9 & 1,5 \\
\hline Zona euro & 10,3 & 1,6 & 1,6 & 1,7 & 0,1 & 0,5 & 1,4 \\
\hline Europa del este & - & 3,4 & 3,0 & 2,8 & $-0,4$ & 0,6 & 2,6 \\
\hline
\end{tabular}

Fuente: Elaboracion propia en base a BNP Paribas (2015)

¿Cómo estamos nosotros? Pues no muy bien. Venezuela, cuya evolución económica e inflacionaria Argentina tendrá una mala performance en términos anticipa la salida del presidente Nicolás Maduro del de crecimiento económico (-1.5\% para 2016) y poder. Por último, es interesante analizar qué es lo inflación elevada para el 2017 (17-0 \%). Colombia que podría ocurrir con los dos gigantes de América no tiene mala performance en términos de creci- latina: Brasil y México. Brasil está registrando una miento económico —aunque sea una tasa baja—, suba de su tasa de inflación con mal nivel de actimientras que se observa una clara tendencia a la vidad económica, mientras que México mantiene desaceleración inflacionaria. Sí está muy mal es ambas variables en torno al 3 \% promedio. 
Gráfico 21. Perspectivas de crecimiento e inflación.

América Latina.

\begin{tabular}{|c|c|c|c|c|c|c|}
\hline \multirow[t]{2}{*}{ PAÍS/ÁREA } & \multicolumn{3}{|c|}{ \% CRECIMIENTO PIB } & \multicolumn{3}{|c|}{ INFLACIÓN } \\
\hline & 2015 & 2016 & 2017 & 2015 & 2016 & 2017 \\
\hline Total Latinoamerica & 0,2 & $-0,1$ & 2,1 & 13,5 & 17,3 & 10,7 \\
\hline Argentina & 1,3 & 0,1 & 3,2 & 23,5 & 32,0 & 17,7 \\
\hline Brasil & $-3,7$ & 3,1 & 0,5 & 10,7 & 7,1 & 5,6 \\
\hline Chile & 2,0 & 2,1 & 2,8 & 4,4 & 3,5 & 2,8 \\
\hline Colombia & 2,9 & 2,6 & 3,1 & 6,8 & 5,0 & 3,6 \\
\hline Ecuador & 0,3 & 0,2 & 1,5 & 3,4 & 2,8 & 3,3 \\
\hline Mexico & 2,5 & 2,8 & 3,1 & 2,1 & 3,7 & 3,3 \\
\hline Peru & 2,9 & 3,4 & 4,4 & 4,4 & 3,3 & 2,9 \\
\hline Venezuela & $-7,6$ & 6,9 & 0,5 & 181,0 & 320,0 & 172,0 \\
\hline Resto Latinoamérica & 4,1 & 3,9 & 3,9 & 2,9 & 4,2 & 4,3 \\
\hline
\end{tabular}

\section{Conclusiones: ¿cómo nos encontramos y qué esperar?}

Vamos a cerrar el documento con una suerte de presentación de lo que son los principales aspectos con los que se podría caracterizar el actual momento económico a nivel mundial:

- Crecimiento mundial mejora levemente pero continúa en niveles moderados.

- Frágil recuperación en la Euro Zona. Países europeos afectados por la crisis, con «pulmotor».

- Incertidumbre acotada por el Brexit.

- Perspectiva benigna de inflación contiene los riesgos de deflación en la Euro Zona pero se revierte en países emergentes.

- Precios de los commoditties primarios con tendencia decreciente en un marco de crecimiento leve del comercio mundial.

- Reducción de los flujos financieros a las economías emergentes aunque con probable reversión a mediano plazo.

- Apreciación del dólar en términos de todas las monedas domésticas.

- Ajustes en las respectivas políticas económicas nacionales corren riesgos moderados de generar inestabilidad macroeconómica (particularmente, a nivel de los grandes países desarrollados).

- Economías emergentes vuelven a enfrentar combinación (más leve que en el pasado) de vulnerabilidad interna + externa.

- Indefinición acotada de la política monetaria de los Estados Unidos (moderándose a largo plazo). La recuperación del apalancamiento financiero y la laxitud monetaria, particularmente la europea, pueden incubar otra crisis.

- Reforma fiscal en la Zona del euro redefine el poder interno y plantea desafíos a nivel doméstico.

- Imperioso fortalecimiento de la coordinación y/0 cooperación de las políticas económicas nacionales a nivel mundial.

Los elementos antes enunciados se inscriben en que la etapa actual del capitalismo avanzado comienza a replantear la vigencia del paradigma socioeconómico y políticos occidental. Su reemplazo no está claro como la sostenibilidad de la dinámica que genere, por lo que la falta de un claro liderazgo mundial a largo plazo 
incentiva la proliferación de conflictos regionales con impacto global. Al mismo tiempo, la duración de la inestabilidad mundial, en un clima de mayor inestabilidad política, racial $y / 0$ religiosa, genera tensiones consistentes que profundizan su impacto negativo a nivel económico y social. En ese marco, el crecimiento mundial continúa siendo modesto y desigual a nivel regional. América Latina y el Caribe enfrentan un escenario pesimista a corto-mediano plazo como a nivel del norte versus el sur de la región.

\section{Referencias bibliográficas}

- BNP Paribas (2015). Informe Internacional 2015. Recuperado de https://invest.bnpparibas.com/en/ internationalreport-2015

- Buchieri, F. (2008). Prestamista de última instancia y cajas de conversión bajo crisis bancarias: «inestabilidad endógena» en un modelo de equilibrio múltiples. Revista Ciencias Económicas, 6(2), 9-28.

- _- (2009). Crisis bancarias recientes en argentina: un modelo teórico y evidencia empírica asociada. Revista Ciencias Económicas, 7(2), 11-21.

- _- (2011). Desarrollo del sistema financiero y crecimiento económico: teoría y evidencia empírica hasta la reciente crisis financiera internacional. Revista Ciencias Económicas, 9(1), 11-39.

- Comisión Económica para América Latina y el Caribe (CEPAL). (2009). La actual crisis financiera internacional y sus efectos en América Latina y el Caribe. Santiago de Chile. Recuperado de http://repositorio.cepal.org/bitstream/handle/11362/2924/S2009328 es.pdf;js essionid=BAFA8B8077FFF5D13E8 BDDBEF970009E? sequence $=1$

- Cerón Cruz, J. (2008). Crisis financieras internacionales, teorías explicativas y propuestas de reforma del Sistema Monetario: el caso de las sub-prime. X Reunión de Economía Mundial. España: Uni- versidad Carlos III. Recuperado de http://e-archivo.uc3m.es/bitstream/ handle/10016/2702/ceron-ponenciacrisis.pdf? sequence $=1$

- Eurostat (2016). Estadísticas. Recuperado de http://ec.europa.eu/ eurostat

- Fernandez-Arias, E., Powell, A., y Rebucci, A. (2009). The Multilateral Response to the Global Crisis: Rationale, Modalities, and Feasibility. BID Research Department Working Papers Nro. 683, Nueva York. Recuperado de https://publications.iadb. org/handle/11319/1653?localeattribute $=$ en

- Fondo Monetario Internacional (FMI). (2014, octubre). Perspectivas de la economía mundial. Crecimiento mundial decepcionante, ritmo de recuperación desigual y diferente en cada país. Revista del Fondo Monetario Internacional. Recuperado de https://www.imf.org/ es/News/Articles/2015/09/28/04/53/ sonew100714a

- Heroles Cardoso, R. (2014). La crisis financiera: orígenes y efectos. Gaceta de Economía, 14(26), 1-13. - Izquierdo, A., y Talvi, E. (2009). Policy Trade-offs for Unprecedented Times. Confronting the Global Crisis in Latin America and the Caribbean. Informe del Banco Interamericadno de Desarrollo. Recuperado de http://www.iadb.org/en/researchand-data/publication-details,3169. html?pub_id=b-635
- Laffaye, S. (2009). Evolución reciente de la economía internacional. Revista de Comercio Exterior e Integración, 14, 51-64. Recuperado de http://www.cei.gov.ar/userfiles/ Evolucion_reciente_de_la_economia_interncional.pdf

- Ocampo A. (2011, agosto). Macroeconomía para el desarrollo: políticas anti-cíclicas y transformación productiva. Revista de la Comisión Económica para América Latina y el Caribe, 104, 7-35.

- _- (2009, abril). Impactos de la crisis financiera mundial sobre América Latina. Revista de la Comisión Económica para América Latina y el Caribe, 97, 9-32.

- SELA (2012, junio). El actual panorama económico mundial y sus implicancias para América Latina y el Caribe. Notas estratégicas, 2. Recuperado de http://www.sela.org/ media/1876666/boletin_notas_estrategicas_no2_espanol.pdf

\section{Registro bibliográfico}

Buchieri, F.E. (2016). La economía mundial amesetada: algunas reflexiones a ocho años de la última crisis financiera global. Revista Ciencias Económicas, 13(02), 89-105. 